\title{
On the imbedding of the Schwarzschild space-time II.
}

By

Tamehiro Fujitani, Mineo Ikeda and Makoto Matsumoto

(Received June 13, 1961)

1. In the previous paper, we obtained the equation (4.3):

(1) $\boldsymbol{z}=\boldsymbol{y}(t, r)+r\left(\sin \theta \sin \varphi \boldsymbol{e}_{4}+\sin \theta \cos \varphi \boldsymbol{e}_{5}+\cos \theta \boldsymbol{e}_{6}\right)$,

which was the general imbedding function of the Schwarzschild space-time $V^{4}$ into a pseudo-euclidean $E^{6}$. The function $y(t, r)$ in (1) should be chosen as satisfying (4.8):

$$
\begin{aligned}
& \left\langle\frac{\partial \boldsymbol{y}}{\partial t}, \frac{\partial \boldsymbol{y}}{\partial t}\right\rangle=\frac{r-2 m}{r},\left\langle\frac{\partial \boldsymbol{y}}{\partial t}, \frac{\partial \boldsymbol{y}}{\partial r}\right\rangle=0, \\
& \left\langle\frac{\partial \boldsymbol{y}}{\partial r}, \frac{\partial \boldsymbol{y}}{\partial r}\right\rangle=-\frac{2 m}{r-2 m} .
\end{aligned}
$$

Conversely, we can easily verify by a direct calculation that the four-dimensional subspace $V^{4}$ in $E^{6}$ defined by (1) has the induced metric which is the same as the one of the Schwarzschild spacetime. The geometrical meaning of (2) is as follows. Since $y$ is a three dimensional vector-valued function of two variables $t$ and $r$, it defines a two-dimensional subspace $V^{2}$ in a pseudo-euclidean $E^{3}$, and the equation (2) shows that the induced metric of the $V^{2}$ is

$$
d s^{2}=\frac{r-2 m}{r} d t^{2}-\frac{2 m}{r-2 m} d r^{2}
$$

Therefore the $y$ may be regarded as the imbedding function of the two-dimensional Riemannian space $V^{2}$ with the indefinite metric (3) into the $E^{3}$. Thus we have 
Theorem 1. The imbedding problem of the Schwarzschild spacetime $V^{4}$ into a pseudo-euclidean space $E^{6}$ is equivalent to the one of a two-dimensional Riemannian space $V^{2}$ with the indefinite metric (3) into a pseudo-euclidean space $E^{3}$. Namely, if $\boldsymbol{y}(t, r)$ is any imbedding function of the $V^{2}$ into an $E^{3}$ with a signature $\varepsilon_{1}+\varepsilon_{2}+\varepsilon_{3}$, then the function (1) gives an imbedding of the Schwarzschild space-time $V^{4}$ into a pseudo-euclidean space $E^{6}$ with the signature $\varepsilon_{1}+\varepsilon_{2}+\varepsilon_{3}-3$, where $\varepsilon_{1}, \varepsilon_{2}$ and $\varepsilon_{3}= \pm 1$. And conversely, any imbedding function of the $V^{4}$ into an $E^{6}$ is obtained by this way.

2. In this paper, we shall give another proof of the theorem. The following method of the proof is closely connected with the one of Kasner's paper [1] and clarifies the freedom of the imbedding.

We now introduce a three-dimensional Riemannian space $V^{3}(t)$ with the positive-definite metric

$$
\begin{aligned}
& d s^{2}=g_{i j} d x^{i} d x^{j}, \quad i, j=1,2,3, \\
& g_{11}=\frac{r}{r-2 m}, \quad g_{22}=r^{2}, \quad g_{33}=r^{2} \sin ^{2} \theta, \\
& g_{i j}=0, \quad i=j, \quad r>2 m,
\end{aligned}
$$

which is obtained from the Schwarzschild metric by putting $t=$ constant and changing the algebraic sign. We shall call the $V^{3}(t)$ the stationary hypersurface in the $V^{4}$. The Christoffel's symbols $\Gamma^{\prime}{ }_{j k}(i, j, k=1,2,3)$ and the curvature tensor $R_{i j k l}(i, j, k, l=1,2,3)$ of the $V^{3}(t)$ are given as follows.

$$
\begin{gathered}
\mathrm{L}_{11}^{11}=-\frac{m}{r(r-2 m)}, \quad \mathrm{I}_{21}^{2}=\mathrm{L}_{12}^{2}=\mathrm{I}_{31}^{3}=\mathrm{I}_{13}^{3}=\frac{1}{r}, \\
\mathrm{I}_{32}^{3}=\mathrm{I}_{23}^{3}=\cot \theta, \quad \mathrm{I}_{22}^{1} \mathrm{l}=-(r-2 m), \\
\mathrm{L}_{33}^{\mathrm{1}}=-(r-2 m) \sin ^{2} \theta, \mathrm{I}_{33}^{2}=-\sin \theta \cos \theta, \text { the other } \mathrm{I}_{j k}^{i}=0, \\
R_{1212}=-\frac{m}{r-2 m}, \quad R_{2323}=2 m r \sin ^{2} \theta, \quad R_{3131}=-\frac{m}{r-2 m} \sin ^{2} \theta,
\end{gathered}
$$

the other $R_{i j k l}=0$.

3. We first consider the stationary $V^{3}(t)$ and shall prove 
Theorem 2. The stationary hypersurface $V^{3}(t)$ in the $V^{4}$ can be imbedded in the euclidean space $E^{4}$ with the positive-definite metric, and then the $V^{3}(t)$ is rigid*.

Proof. The Gauss and Weingarten formulas of $V^{3}(t)$ in an pseudo-enclidean $E^{4}(\mathrm{I}-(1.5))$ are

$$
\begin{gathered}
\nabla_{j} \boldsymbol{u}_{i}=e b_{i j} \boldsymbol{n}, \quad e= \pm 1, \\
\nabla_{j} \boldsymbol{n}=-g^{i k} b_{i j} \boldsymbol{u}_{k},
\end{gathered}
$$

and the Gauss and Codazzi equations (I-(1.7), (1.8)) are

$$
\begin{gathered}
e R_{i j k l}=b_{i k} b_{j l}-b_{i l} b_{j k}, \\
\nabla_{k} b_{i j}-\nabla_{j} b_{i k}=0 .
\end{gathered}
$$

Following the general theory developed by T. Y. Thomas [2], we shall solve the algebraic equation (6). First we get from (6)

$$
\begin{aligned}
{\left[\operatorname{det}\left(b_{i j}\right)\right]^{2} } & =\left|\begin{array}{lll}
e R_{2323} & e R_{2331} & e R_{2312} \\
e R_{3123} & e R_{3131} & e R_{3112} \\
e R_{1223} & e R_{1231} & e R_{1212}
\end{array}\right| \\
& =e R_{2323} R_{3131} R_{1212}=e \frac{2 m^{3} r \sin ^{4} \theta}{(r-2 m)^{2}}>0 .
\end{aligned}
$$

Hence we must take the $\operatorname{sign} e=+1$, and the $b_{i j}$ are given by**

$$
b_{i i}=\frac{R_{i j i j} R_{i k i k}}{\left(R_{2323} R_{3131} R_{1212}\right)^{1 / 2}}, \quad b_{i j}=0, \quad i, j, k \neq .
$$

Thus we have the unique system of solutions of (6) as follows.

$$
\begin{array}{ll}
b_{11}=\frac{m}{(r-2 m)(2 m r)^{1 / 2}}, & b_{22}=-(2 m r)^{1 / 2}, \\
b_{33}=-(2 m r)^{1 / 2} \sin ^{2} \theta, & b_{i j}=0, \quad i \neq j .
\end{array}
$$

By substitution from (8) into (7), we see easily that these (8) satisfy (7) automatically. Therefore the $V^{3}(t)$ under consideration can be imbedded in the euclidean $E^{4}$. It follows from $\operatorname{det}\left(b_{i j}\right) \neq=0$ that the hypersurface $V^{3}(t)$ in $E^{4}$ is of type three in the sense of T. Y. Thomas, and hence we have the rigidity of the $V^{3}(t)$ regarded as a hypersurface of the $E^{4}$.

* See [5] of [2]. ** See $(9.4)$ of [2]. 
4. Next, we shall find the imbedding function $\boldsymbol{u}$ of the $V^{3}(t)$ into $E^{4}$. The Gauss formula (4) is now rewritten in the forms

$$
\begin{aligned}
& \frac{\partial^{2} \boldsymbol{u}}{\partial r^{2}}=-\frac{m}{r(r-2 m)} \frac{\partial \boldsymbol{u}}{\partial r}+\underset{(r-2 m)(2 m r)^{1 / 2} \boldsymbol{n},}{\frac{\partial^{2} \boldsymbol{u}}{\partial \theta^{2}}}=-(r-2 m) \frac{\partial \boldsymbol{u}}{\partial \boldsymbol{r}}-(2 m r)^{1 / 2} \boldsymbol{n}, \\
& \frac{\partial^{2} \boldsymbol{u}}{\partial \varphi^{2}}=-(r-2 m) \sin ^{2} \theta \frac{\partial \boldsymbol{u}}{\partial r}-\sin \theta \cos \theta \frac{\partial \boldsymbol{u}}{\partial \theta}-(2 m r)^{1 / 2} \sin ^{2} \theta \boldsymbol{n}, \\
& \frac{\partial^{2} \boldsymbol{u}}{\partial r \partial \theta}=\frac{1}{r} \frac{\partial \boldsymbol{u}}{\partial \theta}, \quad \frac{\partial^{2} \boldsymbol{u}}{\partial \theta \partial \rho}=\cot \theta \frac{\partial \boldsymbol{u}}{\partial \varphi}, \quad \frac{\partial^{2} \boldsymbol{u}}{\partial r \partial \rho}=\frac{1}{r} \frac{\partial \boldsymbol{u}}{\partial \rho},
\end{aligned}
$$

and the Weingarten formula (5) is expressed in the forms

$$
\begin{aligned}
& \frac{\partial \boldsymbol{n}}{\partial \boldsymbol{r}}=-\frac{(2 m \boldsymbol{r})^{1 / 2}}{2 r^{2}} \frac{\partial \boldsymbol{u}}{\partial \boldsymbol{r}}, \\
& \frac{\partial \boldsymbol{n}}{\partial \theta}=\frac{(2 m r)^{1 / 2}}{r^{2}} \frac{\partial \boldsymbol{u}}{\partial \theta}, \\
& \frac{\partial \boldsymbol{n}}{\partial \varphi}=\frac{(2 m \boldsymbol{r})^{1 / 2}}{r^{2}} \frac{\partial \boldsymbol{u}}{\partial \varphi}
\end{aligned}
$$

Furthermore, the imbedding function $\boldsymbol{u}$ should satisfy the isometry conditions :

$$
\begin{aligned}
& \left\langle\frac{\partial \boldsymbol{u}}{\partial r}, \frac{\partial \boldsymbol{u}}{\partial r}\right\rangle=\frac{r}{r-2 m},\left\langle\frac{\partial \boldsymbol{u}}{\partial \theta}, \frac{\partial \boldsymbol{u}}{\partial \theta}\right\rangle=r^{2} \\
& \left\langle\frac{\partial \boldsymbol{u}}{\partial \varphi}, \frac{\partial \boldsymbol{u}}{\partial \mathscr{P}}\right\rangle=r^{2} \sin ^{2} \theta \\
& \left\langle\frac{\partial \boldsymbol{u}}{\partial r}, \frac{\partial \boldsymbol{u}}{\partial \theta}\right\rangle=\left\langle\frac{\partial \boldsymbol{u}}{\partial r}, \frac{\partial \boldsymbol{u}}{\partial \mathscr{P}}\right\rangle=\left\langle\frac{\partial \boldsymbol{u}}{\partial \theta}, \frac{\partial \boldsymbol{u}}{\partial \mathscr{P}}\right\rangle=0,
\end{aligned}
$$

and the normal $\boldsymbol{n}$ should accept the normality conditions:

$$
\begin{aligned}
& \left\langle\frac{\partial \boldsymbol{u}}{\partial \boldsymbol{r}}, \boldsymbol{n}\right\rangle=\left\langle\frac{\partial \boldsymbol{u}}{\partial \theta}, \boldsymbol{n}\right\rangle=\left\langle\frac{\partial \boldsymbol{u}}{\partial \varphi}, \boldsymbol{n}\right\rangle=0, \\
& \langle\boldsymbol{n}, \boldsymbol{n}\rangle=1 .
\end{aligned}
$$

The solutions $\boldsymbol{u}$ and $\boldsymbol{n}$ of the equations (9) and (10) are easily obtained by

(13) $\boldsymbol{u}=-2[2 m(\boldsymbol{r}-2 m)]^{1 / 2} \boldsymbol{e}_{1}+r \sin \theta\left(\sin \boldsymbol{P} \boldsymbol{e}_{4}+\cos \boldsymbol{P} \boldsymbol{e}_{5}\right)+r \cos \theta \boldsymbol{e}_{6}$, 
(14) $\boldsymbol{n}=\left(\frac{r-2 m}{r}\right)^{1 / 2} \boldsymbol{e}_{1}+\frac{(2 m r)^{1 / 2}}{r} \sin \theta\left(\sin \phi \boldsymbol{e}_{4}+\cos \varphi \boldsymbol{e}_{5}\right)+\cos \theta \boldsymbol{e}_{6}$.

Then the equations (11) and (12) imply that the $\boldsymbol{e}_{1}, \boldsymbol{e}_{4}, \boldsymbol{e}_{5}$ and $\boldsymbol{e}_{6}$ are orthonormal unit vectors.

5. We now return to the Schwarzschild space-time $V^{4}$. Its metric is expressed by

$$
d s^{2}=\frac{r-2 m}{r} d t^{2}-\left(d s^{2} \text { of the } V^{3}(t)\right),
$$

and so the equation (13) shows that the Schwarzschild metric $d s^{2}$ is written in the form

$$
d s^{2}=\frac{r-2 m}{r} d t^{2}-(d y)^{2}-\left(d z_{4}\right)^{2}-\left(d z_{5}\right)^{2}-\left(d z_{6}\right)^{2},
$$

where we put

$$
\begin{aligned}
& y=-2[2 m(r-2 m)]^{1 / 2}, \quad z_{4}=r \sin \theta \sin \mathcal{P}, \\
& z_{5}=r \sin \theta \cos \varphi, \quad z_{6}=r \cos \theta .
\end{aligned}
$$

The equation (15) is equivalent to the equation (4) of Kasner's paper [1]. The $y$ is the function of $r$ only and we have

$$
\frac{r-2 m}{r} d t^{2}-(d y)^{2}=\frac{r-2 m}{r} d t^{2}-\frac{2 m}{r-2 m} d r^{2} \text {. }
$$

which is the same as (3). Therefore we have proved Theorem 1 .

6. Consequently we may say that our imbedding problem of the Schwarzschild space-time $V^{4}$ is reduced to the one of the $V^{2}$ with the metric (3) into an $E^{3}$. The Christoffel's symbols $\mathrm{I}_{j k}^{i}(i, j, k=0,1)$ of the $V^{2}$ are given by

$$
\begin{aligned}
& \Lambda_{00}^{10}=\Lambda_{01}^{1}=\Lambda_{11}^{0}=0, \quad \Lambda_{00}^{1}=\frac{r-2 m}{2 r^{2}}, \\
& \Lambda_{01}^{10}=\frac{m}{r(r-2 m)}, \quad \Lambda_{11}^{11}=-\frac{1}{2(r-2 m)},
\end{aligned}
$$

and the curvature tensor of the $V^{2}$ is

$$
R_{0101}=\frac{3 m}{2 r^{3}}
$$


Hence the Gauss and Codazzi equations of the $V^{2}$ in the $E^{3}$ are written in the forms

$$
\begin{aligned}
& b_{00} b_{11}-\left(b_{01}\right)^{2}=\frac{3 m}{2 r^{3}} \varepsilon, \quad \varepsilon= \pm 1 \\
& \frac{\partial b_{00}}{\partial r}-\frac{\partial b_{01}}{\partial t}-\frac{m}{r(r-2 m)} b_{00}+\frac{r-2 m}{2 r^{2}} b_{11}=0 \\
& \frac{\partial b_{01}}{\partial r}-\frac{\partial b_{11}}{\partial t}+\frac{r+2 m}{2 r(r-2 m)} b_{01}=0 .
\end{aligned}
$$

The imbedding function $\boldsymbol{y}$ and the normal $\boldsymbol{m}$ are the solutions of the Gauss and Weingarten formulas as follows:

$$
\begin{aligned}
& \left\{\begin{array}{l}
\frac{\partial^{2} \boldsymbol{y}}{\partial t^{2}}=\frac{r-2 m}{2 r^{2}} \frac{\partial \boldsymbol{y}}{\partial r}+b_{00} \boldsymbol{m} \\
\frac{\partial^{2} \boldsymbol{y}}{\partial t \partial r}=\frac{m}{r(r-2 m)} \frac{\partial \boldsymbol{y}}{\partial t}+b_{01} \boldsymbol{m}, \\
\frac{\partial^{2} \boldsymbol{y}}{\partial r^{2}}=-\frac{1}{2(r-2 m)} \frac{\partial \boldsymbol{y}}{\partial r}+b_{11} \boldsymbol{m}
\end{array}\right. \\
& \left\{\begin{array}{l}
\frac{\partial \boldsymbol{m}}{\partial t}=-\frac{r}{r-2 m} b_{00} \frac{\partial \boldsymbol{y}}{\partial t}+\frac{r-2 m}{2 m} b_{01} \frac{\partial \boldsymbol{y}}{\partial r} \\
\frac{\partial \boldsymbol{m}}{\partial r}=-\frac{r}{r-2 m} b_{01} \frac{\partial \boldsymbol{y}}{\partial t}+\frac{r-2 m}{2 m} b_{11} \frac{\partial \boldsymbol{y}}{\partial r}
\end{array}\right.
\end{aligned}
$$

The three-dimensional vectors $\boldsymbol{y}$ and $\boldsymbol{m}$ satisfy the well-known algebraic conditions :

$$
\begin{aligned}
& \left\{\begin{array}{l}
\left\langle\frac{\partial \boldsymbol{y}}{\partial r}, \frac{\partial \boldsymbol{y}}{\partial r}\right\rangle=-\frac{2 m}{r-2 m},\left\langle\frac{\partial \boldsymbol{y}}{\partial r}, \frac{\partial \boldsymbol{y}}{\partial t}\right\rangle=0, \\
\left\langle\frac{\partial \boldsymbol{y}}{\partial t}, \frac{\partial \boldsymbol{y}}{\partial t}\right\rangle=\frac{r-2 m}{r},
\end{array}\right. \\
& \left\{\begin{array}{l}
\left\langle\frac{\partial \boldsymbol{y}}{\partial r}, \boldsymbol{m}\right\rangle=\left\langle\frac{\partial \boldsymbol{y}}{\partial t}, \boldsymbol{m}\right\rangle=0, \\
\langle\boldsymbol{m}, \boldsymbol{m}\rangle=1,
\end{array}\right.
\end{aligned}
$$

where the symbol $\langle$,$\rangle is the inner product in the E^{3}$. As is wellknown, a system of solutions $b_{i j}$ of (19) and (20) corresponds oneto-one to an imbedding function $y$ of the $V^{2}$ (within a motion in the $E^{3}$ ) and hence to an imbedding function $z$ of the Schwarzschild space time $V^{4}$. 
7. For example, we consider the case where $b_{00}, b_{01}$ and $b_{11}$ in (19) and (20) do not depend upon $t$. These $b_{i j}$ are immediately given by

$$
\begin{aligned}
& \left(b_{00}\right)^{2}=\frac{m(r-2 m)}{2 r^{4}}+\frac{c^{2}}{r}+\frac{c^{\prime}(r-2 m)}{r}, \\
& b_{01}=\frac{c(r)^{1 / 2}}{r-2 m}, \\
& b_{11}=\frac{1}{b_{00}}\left(\frac{3 m}{2 r^{3}}+\frac{c^{2} r}{(r-2 m)^{2}}\right),
\end{aligned}
$$

where $c$ and $c^{\prime}$ are integral constants. On the other hand, we have obtained the stationary solutions at the end of the previous paper. If we deal with the stationary solution (i), then we have

$$
\left(d y_{1}\right)^{2}+\left(d y_{2}\right)^{2}-\left(d y_{3}\right)^{2}=\frac{r-2 m}{r} d t^{2}-\frac{2 m}{r-2 m} d r^{2},
$$

from which it follows that

$$
\begin{aligned}
& \frac{\partial \boldsymbol{y}}{\partial t}=\left(\gamma^{1 / 2} \cos \frac{t}{k},-\gamma^{1 / 2} \sin \frac{t}{k}, 0\right), \\
& \frac{\partial^{2} \boldsymbol{y}}{\partial t^{2}}=\left(-\frac{\gamma^{1 / 2}}{k} \sin \frac{t}{k},-\frac{\gamma^{1 / 2}}{k} \cos \frac{t}{k}, 0\right), \\
& \frac{\partial^{2} \boldsymbol{y}}{\partial t \partial r}=\left(\frac{m}{r^{2} \gamma^{1 / 2}} \cos \frac{t}{k},-\frac{m}{r^{2} \gamma^{1 / 2}} \sin \frac{t}{k}, 0\right), \\
& \boldsymbol{m}=\left(p \sin \frac{t}{k}, p \cos \frac{t}{k}, p \frac{k m}{r^{2} \gamma^{1 / 2} f^{\prime}}\right),
\end{aligned}
$$

where we put

$$
\gamma=\frac{r-2 m}{r}, \quad p^{2}=1+\frac{m k^{2}}{2 r^{3}} .
$$

Hence the equation (21) gives

$$
b_{01}=0, \quad\left(b_{00}\right)^{2}=\frac{r-2 m}{r}\left(\frac{m}{2 r^{3}}+\frac{1}{k^{2}}\right) .
$$

These are obtained from (25), if we take $c=0$ and $c^{\prime}=\frac{1}{k^{2}}$. Similarly we can verify that the other stationary solutions (ii) and (iii) 


\section{Tamehiro Fujitani, Mineo Ikeda and Makoto Matsumoto}

are special cases of (25), the former being $c=0, c^{\prime}=-\frac{1}{k^{2}}$, and the the later being $c=0, c^{\prime}=0$. Thus we see that the stationary solutions in the previous paper are the special cases of the solutions (25), where we put $c=0$.

University of Osaka Prefecture, The Research Institute for Theoretical Physics, Hiroshima University, and Institute of Mathematics, Kyoto University.

\section{REFERENCES}

[1] E. Kasner: Finite representation of the solar gravitational field in flat space of six-dimensions. Amer. J. Math. 43 (1921), 130-133.

[2] T. Y. Thomas: Riemann spaces of class one and their characterizations. Acta. Math. 67 (1936), 169-211. 